\title{
Fatores de risco para a síndrome de burnout em profissionais da saúde
}

\author{
Risk factors for burnout syndrome in healthcare professionals
}

Factores de riesgo del síndrome de burnout en profesionales sanitarios

Yasmin Lucena Dantas ${ }^{1 *}$, Nathália de Oliveira Azevedo ${ }^{1}$, Mayrlla Myrelly Vieira Formiga ${ }^{1}$, Daniel Gustavo Guedes Pereira de Albuquerque ${ }^{1}$, Ana Clara Amorim Noronha1', Lívia de Castro Mestre ${ }^{1}$, João Assis Herculano Araruna1, Fabiana Medeiros de Brito' ${ }^{1}$.

\section{RESUMO}

Objetivo: Analisar os fatores de risco para a síndrome de burnout em profissionais da saúde. Métodos: Trata-se de uma pesquisa descritiva, transversal, com abordagem quantitativa, realizada em um hospital público. A população foi composta por uma equipe multiprofissional da Unidade de Terapia intensiva (UTI) do referido serviço, com uma amostra composta por 73 profissionais. Resultados: A pesquisa obteve maior prevalência de técnicos de enfermagem (43,8\%), seguidos por fisioterapeutas (20,5\%), enfermeiros (19,2\%) e médicos $(15,1 \%)$, com associação estatisticamente significativa para risco de burnout. A maioria dos profissionais são do sexo feminino $(65,8 \%)$, possuem entre 30 e 39 anos $(37,0 \%)$, têm filhos $(56,2 \%)$, são casados $(46,6 \%)$, atuam na área há mais de 10 anos $(45,2 \%)$, recebem até 5 salários mínimos $(79,4 \%)$, com jornada de trabalho semanal $(68,5 \%)$ de 30 horas, trabalhando em mais de um hospital $(78,1 \%)$, de maneira integral $(76,7 \%)$. Conclusão: A identificação de tais fatores de risco é de extrema importância para a saúde dos trabalhadores da saúde, oferecendo subsídios a adoção de medidas preventivas e enfrentamento do referido problema.

Palavras-chave: Burnout, Profissionais da saúde, Fatores de risco.

\begin{abstract}
Objective: To analyze risk factors for burnout syndrome in health professionals. Methods: This is a descriptive, cross-sectional research with a quantitative approach, carried out in a public hospital. The population consisted of a multidisciplinary team from the Intensive Care Unit (ICU) of that service, with a sample of 73 professionals. Results: The survey had a higher prevalence of nursing technicians (43.8\%), followed by physical therapists (20.5\%), nurses (19.2\%) and physicians (15.1\%), with a statistically significant association for risk of burnout. Most professionals are female $(65.8 \%)$, are between 30 and 39 years old $(37.0 \%)$, have children $(56.2 \%)$, are married $(46.6 \%)$, have been working in the field for a long time, over 10 years $(45.2 \%)$, receive up to 5 minimum wages $(79.4 \%)$, with a weekly working day (68.5\%) of 30 hours, working in more than one hospital $(78.1 \%)$, in full $(76.7 \%)$. Conclusion: The identification of such risk factors is extremely important for the health of health workers, offering support for the adoption of preventive measures and coping with the aforementioned problem.
\end{abstract}

Keywords: Burnout, Health professionals, Risk factors.

\section{RESUMEN}

Objetivo: Analizar los factores de riesgo del síndrome de burnout en profesionales de la salud. Métodos: Se trata de una investigación descriptiva, transversal con enfoque cuantitativo, realizada en un hospital público. La población estuvo conformada por un equipo multidisciplinario de la Unidad de Cuidados Intensivos (UCl) de ese servicio, con una muestra de 73 profesionales. Resultados: La encuesta tuvo una mayor prevalencia de técnicos de enfermería $(43,8 \%)$, seguida de fisioterapeutas $(20,5 \%)$, enfermeras $(19,2 \%)$ y médicos $(15,1 \%)$, con asociación estadísticamente significativa para el riesgo de burnout. La mayoría de los profesionales son mujeres (65,8\%), tienen entre 30 y 39 años $(37,0 \%)$, tienen hijos $(56,2 \%)$,

\footnotetext{
${ }^{1}$ Faculdade de Ciências Médicas da Paraíba (FCMPB), João Pessoa - PB.

*E-mail: yasdanttas97@gmail.com
} 
están casados $(46,6 \%)$, llevan mucho tiempo trabajando en el campo. Más de 10 años $(45,2 \%)$, perciben hasta 5 salarios mínimos $(79,4 \%)$, con una jornada semanal $(68,5 \%)$ de 30 horas, laborando en más de un hospital $(78,1 \%)$, en su totalidad (76,7\%). Conclusión: La identificación de dichos factores de riesgo es de suma importancia para la salud de los trabajadores de la salud, ofreciendo apoyo para la adopción de medidas preventivas y el afrontamiento del problema mencionado.

Palabras clave: Burnout, Profesionales de la salud, Factores de riesgo.

\section{INTRODUÇÃO}

A repercussão do trabalho na saúde física e, sobretudo, mental dos profissionais tem sido cada vez mais estudada nos últimos anos, visto que estudos já atestam os efeitos negativos de longos turnos de trabalho na qualidade de vida e no desempenho das atividades laborais (ELDEVIK MF, et al., 2013). Lima RAS, et al. (2013) frisa que o trabalho nem sempre é fonte de realização pessoal e profissional, podendo este, gerar impasses como exaustão e insatisfação.

Fatores como condições indevidas de trabalho, complexidade de procedimentos, remuneração diminuta, estresse e exposição a riscos destacam-se como consequências negativas no que se refere ao bem-estar dos trabalhadores (ROSEINSTEI AH, 2019). Principalmente em relação aos profissionais de saúde e a interferência da saúde mental na segurança do paciente, apontando, assim, para o conceito de desgaste dentre estes (CORREDOR LH, et al., 2016).

Sabe-se que nessa área de atuação os cuidados com a saúde impõem diversas pressões sobre os trabalhadores, incluindo os desafios das atividades clínicas, demandas concorrentes, restrições de tempo, pouco controle sobre os agendamentos e processos de trabalho e relacionamentos conflitantes com a liderança (GARCIA CL, et al., 2019). Além disso, trata-se de ambientes sujeitos a intensos estímulos emocionais na perspectiva de que o objeto de trabalho é um ser enfermo, e que, muitas vezes, pela proximidade com o paciente, acabam a estabelecer vínculos afetivos (SANTOS MA e HORMANEZ M, 2013).

Dessa forma, esse conjunto de conflitos gera um quadro de estresse crônico, o qual traz consequências negativas tanto para a saúde, quanto socioeconômicas. No que concerne a esse panorama conflituoso, surgem enfermidades decorrentes do estresse ocupacional, dentre as quais destaca-se a síndrome de burnout (VIEIRA I e RUSSO JA, 2019).

Esta é definida como uma síndrome psicológica de cunho tridimensional composta por despersonalização, exaustão emocional e realização pessoal a qual ocorre em decorrência de estresse laboral crônico (MASLACH C e LEITER MP, 2016). Essa despersonalização acarreta o desenvolvimento de atitudes negativas, a exaustão causa um dano emocional significativo e a falta de realização pessoal caracteriza-se pela redução dos sentimentos de competência e realização no trabalho (LEITER MP e MASLACH C, 2016).

A 11를 Revisão da Classificação Internacional de Doenças (CID-11) inclui a síndrome de burnout como um fenômeno ocupacional que acarreta danos para a saúde do profissional devido um estresse de caráter crônico no ambiente de trabalho que não foi manejado com êxito. Esta é descrita no capítulo "Fatores que influenciam o estado de saúde ou o contato com os serviços de saúde" e caracteriza sua tridimensionalidade descrevendo o esgotamento de energia; o aumento do distanciamento mental do próprio trabalho e diminuição da eficácia profissional (OPAS, 2019).

Nesse contexto, Perniciotti $P$, et al. (2020) elenca as principais consequências da síndrome de burnout nos profissionais de saúde, a saber: distúrbios individuais, como transtorno de estesse pós-traumático, sintomas psicossomáticos, abuso de substâncias alcoólicas e drogas, ideação suicida e depressão; mudanças comportamentais como insatisfação e intenção de abandono do trabalho, bem como falta de comprometimento com a estrutura organizacional do serviço; problemas no trabalho como absenteísmo, erros de conduta e no que concerne às práticas de segurança; além de implicações para os hospitais como rotatividade de funcionários e aumento dos custos internos. 
Nos últimos anos a incidência de burnout vem aumentando e têm sido realizados numerosos estudos com o objetivo de reconhecer os fatores associados, as causas e as consequências que a síndrome acarreta. Nessa perspectiva, o presente estudo tem o objetivo de analisar os fatores de risco para a síndrome de burnout em profissionais da saúde.

\section{MÉTODOS}

Trata-se de uma pesquisa com alcance descritivo, transversal, com abordagem quantitativa, realizada em um hospital público, localizado no município de João Pessoa-PB. A população selecionada foi composta por uma equipe multiprofissional (dentistas, enfermeiros, fisioterapeutas, médicos e técnicos de enfermagem) da Unidade de Terapia intensiva (UTI) do referido serviço.

A amostra foi do tipo não probabilística, por acessibilidade, seguindo os seguintes critérios de inclusão: profissionais de ambos os sexos, com mais de um ano de atuação no referido local, que estivessem desenvolvendo suas atividades laborais no momento de coleta de dados, e que após devidamente esclarecidos dos objetivos propostos, aceitaram participar da pesquisa assinando o Termo de Consentimento Livre e Esclarecido (TCLE). Desse modo, a amostra foi composta por 73 profissionais.

A coleta de dados ocorreu entre os meses de dezembro de 2018 e fevereiro de 2019, por meio de entrevista, subsidiada por um instrumento estruturado subdividido em duas seções. A primeira seção contemplou itens referentes à caracterização sociodemográfica dos participantes do estudo. Já a segunda seção envolveu aspectos atrelados à identificação dos fatores de risco para síndrome de burnout. Para nortear tal identificação, utilizou-se o Cuestionario para la Evaluación del Síndrome de Quemarse por el Trabajo - CESQT.

O CESQT é um instrumento cujo propósito é a identificação da vulnerabilidade dos indivíduos para o desenvolvimento da síndrome de burnout. Este é validado, autoaplicável, com versão adaptada para uso no Brasil que tem como proposta superar dificuldades psicométricas de outros instrumentos (GIL-MONTE PR, et al., 2010). Este apresenta 20 itens, sendo distribuídos em quatro subescalas ou dimensões: llusão ao trabalho; Desgaste psíquico; Indolência e Culpa. Tais dimensões supracitadas são mensuradas de acordo com a frequência das respostas dos participantes, segundo escala tipo likert, descrita a seguir: 0 (nunca), 1 (raramente), 2 (as vezes: algumas vezes por mês), 3 (frequentemente: algumas vezes por semana) e 4 (diariamente).

Ao final, realiza-se um somatório dos escores das dimensões do CESQT, podendo emergir os seguintes resultados para vulnerabilidade à síndrome de burnout: 1) muito baixo ( $\leq 10)$; 2) baixo (11-33); 3) médio (34$66)$; 4) alto (67-89) e 5) crítico ( $\geq 90)$.

Os dados foram analisados por meio de estatística descritiva através de frequências absolutas e relativas, e para comparação das principais variáveis categóricas foi utilizado o Teste do Qui-quadrado. Para tanto, utilizou-se do Software Microsoft Excel 2016 e do sistema computacional Statistical Package for the Social Sciences - SPSS versão 20.0.

Ressalta-se, ainda, que durante o processo da pesquisa, foram observados os preceitos éticos que normatizam a pesquisa envolvendo seres humanos, conforme rege a Resolução 466/2012 do CNS/MS/BRASIL, sendo o projeto aprovado pelo Comitê de Ética da Faculdade de Ciências Médicas da Paraíba, sob o Protocolo oㅜ 2.493.546/2018 e CAAE no 82901118.3.0000.5178.

\section{RESULTADOS}

No que se refere à caracterização da amostra $(n=73)$, verificou-se que a maioria dos profissionais pertencia ao sexo feminino, $65,8 \%(n=48)$, com faixa etária predominante 30 a 39 anos, correspondendo a $37 \%(n=27) .56,2 \%$ com Filhos $(n=41)$. Quanto à formação acadêmica, 43,8\% $(n=32)$ eram técnicos de enfermagem, 20,5\% fisioterapeutas ( $n=15), 19,2 \%$ enfermeiros $(n=14), 15,1 \%$ médicos $(n=11)$ e 1,4\% dentista $(n=01)$. Além disso, $46,6 \%(n=34)$ casados, seguidos de solteiros com $35,6 \%(n=26)$. E no tocante à renda, 39,7\% recebem até 02 salários mínimos $(n=29), 39,7$ entre 03 e 05 salários mínimos $(n=29)$, recebem de 06 a 09 salários $11 \%(n=08)$ e 10 ou mais salários $9,6 \%(n=07)$. Conforme disposto na Tabela 1. 
Tabela 1 - Distribuição das características sociodemográficas dos profissionais entrevistados $(n=73)$.

\begin{tabular}{|c|c|c|}
\hline Dados sociodemográficos & $\mathbf{n}$ & $\%$ \\
\hline \multicolumn{3}{|l|}{ Sexo } \\
\hline Feminino & 48 & $65,8 \%$ \\
\hline Masculino & 25 & $34,2 \%$ \\
\hline \multicolumn{3}{|l|}{ Faixa etária } \\
\hline 30 a 39 anos & 27 & $37,0 \%$ \\
\hline 20 a 29 anos & 21 & $28,8 \%$ \\
\hline 40 a 49 anos & 16 & $21,9 \%$ \\
\hline Acima de 50 anos & 09 & $12,3 \%$ \\
\hline \multicolumn{3}{|l|}{ Filhos } \\
\hline Sim & 41 & $56,2 \%$ \\
\hline Não & 32 & $43,8 \%$ \\
\hline \multicolumn{3}{|l|}{ Formação acadêmica } \\
\hline Técnico de enfermagem & 32 & $43,8 \%$ \\
\hline Fisioterapeuta & 15 & $20,5 \%$ \\
\hline Enfermeiro & 14 & $19,2 \%$ \\
\hline Médico & 11 & $15,1 \%$ \\
\hline Dentista & 01 & $1,4 \%$ \\
\hline \multicolumn{3}{|l|}{ Estado civil } \\
\hline Casado (a) & 34 & $46,6 \%$ \\
\hline Solteiro (a) & 26 & $35,6 \%$ \\
\hline União estável & 06 & $8,2 \%$ \\
\hline Separado (a) & 04 & $5,5 \%$ \\
\hline Viúvo (a) & 03 & $4,1 \%$ \\
\hline \multicolumn{3}{|l|}{ Renda } \\
\hline Até 2 salários mínimos & 29 & $39,7 \%$ \\
\hline 3 a 5 salários mínimos & 29 & $39,7 \%$ \\
\hline 6 a 9 salários mínimos & 08 & $11,0 \%$ \\
\hline 10 ou mais salários mínimos & 07 & $9,6 \%$ \\
\hline
\end{tabular}

Fonte: Dantas YL, et al., 2021.

Com relação à identificação dos fatores de risco para a síndrome de burnout, quanto ao tempo de atuação na referida profissão, verificou-se que a maioria dos profissionais $(45,2 \%)$ atuam na área há mais de 10 anos. Já no tocante à jornada de trabalho semanal, apontou-se que 50 (68,5\%) dos entrevistados trabalham 30 horas por semana. O presente estudo apontou ainda a prevalência de profissionais trabalhando em mais de um hospital $(78,1 \%)$. A respeito disso, salienta-se que a maior parte destas é de origem privada $(39,7 \%)$.

Com relação ao turno de trabalho, demonstrou-se que a maioria dos participantes trabalham de maneira integral $(76,7 \%)$, seguido de turno diurno $(20,5 \%)$.

No tocante a associação entre a titulação dos participantes e o escore total do CESQT que aponta os riscos para ocorrência de burnout, demonstrou-se relação estatisticamente significativa entre tais variáveis (Tabela 2). 
Tabela 2 - Distribuição e associação entre a formação acadêmica e as dimensões do CESQT (n=73).

\begin{tabular}{lcccc}
\hline \multirow{2}{*}{ Titulação } & \multicolumn{3}{c}{ Escore total do CESQT } & \multirow{2}{*}{ Valor-p \% } \\
\cline { 2 - 4 } & Nível baixo & Nível médio & Nível alto & \\
\hline Técnico & 04 & 26 & - & \\
Graduação & 04 & 14 & - & \\
Especialização & 05 & 16 & - & $<0,045^{\star}$ \\
Mestrado & 01 & 04 & 01 & \\
Doutorado & - & 02 & - & \\
\hline
\end{tabular}

Legenda: *Teste Qui-quadrado. Valor- $p<0,05$.

Fonte: Dantas YL, et al., 2021.

\section{DISCUSSÃO}

No presente trabalho, quando analisada a identificação dos fatores sociodemográficos, verificou-se que a maioria dos profissionais $(65,8 \%)$ são do sexo feminino (Tabela 1). Um estudo descritivo, transversal e multicêntrico também obteve uma amostra de profissionais da saúde predominantemente feminina $(76,2 \%)$ GIL-MONTE PR, et al., 2010). Resultados semelhantes foram encontrados em outros estudos realizados no Brasil e em Portugal (SILVA SCPS, et al., 2015; SOUSA VFS e ARAUJO TCCF, 2015; BORGES EMN, et al., 2019; MARQUES GLC, et al., 2018).

Um trabalho que verifica a incidência de burnout apenas em profissionais de enfermagem, a prevalência feminina dentre os profissionais também foi observada, correspondendo a $87,1 \%$ da amostra, as quais variavam entre 21 e 60 anos (CAMPOS ICM, et al., 2016). Do mesmo modo, em outro estudo houve um predomínio de $77,78 \%$ do gênero (WORM FA, et al., 2016). Como a maioria dos entrevistados neste estudo foram profissionais de enfermagem, os dados sociodemográficos de sexo ratificam essa prevalência.

Possivelmente, essa prevalência feminina demonstra uma tendência da profissão e deve-se, principalmente, ao processo social e histórico que associa a figura da mulher às práticas do cuidado (SOUSA, VFS e ARAUJO TCCF, 2015; CAMPOS ICM, et al., 2015).

Já o estudo para investigar a presença de burnout entre os médicos intensivistas com idade média de 38,7 anos (DP $=8,39$ ) em Sergipe houve uma leve predominância do sexo masculino $(50,8 \%)$ (BARROS MMS, et al., 2016). Da mesma forma em um estudo que analisava a prevalência de síndrome de burnout entre os médicos anestesiologistas, em que foi verificado uma predominância de homens (65,6\%). Dados consistentes com os apresentados pelo Conselho Regional de Medicina do Estado de São Paulo (CREMESP) em 2018, que atribui uma maioria do sexo masculino $(54,4 \%)$ às práticas exclusivamente médicas, com essa diferença caindo ano a ano devido a uma crescente feminização da medicina no país (SCHEFFER, M. et al., 2018).

Analisando os resultados referentes ao sexo predominante encontrado neste trabalho, estudos indicam que o sexo feminino, em geral, possui mais riscos de adquirir a síndrome de burnout devido a dupla presença de mulheres em casa e no trabalho associado a fatores socioeconômicos, como salários mais baixos e/ou maiores demandas no trabalho (OIT, 2016; CAÑADAS-DE LA FUENTE GA, et al., 2015). Outro fato observado em um estudo é que as mulheres apresentam médias superiores aos dos homens quando analisadas sob a óptica do estresse traumático. Esse dado foi associado a maior capacidade empática das mulheres a se relacionar com seus pacientes e absorverem os seus medos e traumas (BORGES EMN, et al., 2019; STAMM BH, 2010).

Com relação à faixa etária predominante, o presente estudo verificou que a maioria dos entrevistados $(37,0 \%)$ estão entre 30 e 39 anos (Tabela 1). Em outros estudos, verificou-se uma média de 37,3 anos $(\mathrm{DP}=8,56)$ dos entrevistados (CAMPOS ICM, et al., 2015) e 37,1 anos ( $\mathrm{DP}=6,3)$ (BORGES EMN, et al., 2019), estando esses resultados dentre a faixa etária verificada. Porém, em outros foram encontradas predominâncias de idade diferentes (GIL-MONTE PR, et al., 2010; SILVA SCPS, et al., 2015; SOUSA, VFS e ARAUJO TCCF, 2015). 
Em um estudo transversal, descritivo e quantitativo, a faixa etária observada para a síndrome de burnout foi de 30 e 50 anos $(64,2 \%)$, no qual sugeriu que os profissionais com maior risco para surgimento do burnout são aqueles com pouca maturidade profissional e, portanto, com menor domínio de emoções em situações de estresse e desgaste profissional (MAGALHÃES E, et al., 2015).

Nesta análise dos fatores sociodemográficos quanto ao estado civil, a predominância foi de profissionais casados (46,6\%) (Tabela 1), dado similar observado em outros trabalhos (SILVA SCPS, et al., 2015; SOUSA, VFS e ARAUJO TCCF, 2015). Já com relação a descendência, este estudo constatou uma prevalência de $56,2 \%$ dos profissionais com filhos. Esse dado foi verificado e associado a profissionais com estado civil casado em outros trabalhos (MAGALHÃES E, et al., 2015).

Quanto a estas variáveis intimamente relacionadas, um estudo relatou a condição de compromisso matrimonial como mecanismo de proteção a doenças físicas e psíquicas, superando positivamente a carga de trabalho/casa (GRILLO LP, et al., 2018). Por outro lado, outros estudos afirmam que a sobrecarga da relação trabalho/casa é uma das principais fontes de estresse e de vulnerabilidade a acidentes de trabalho, estando os solteiros menos susceptíveis ao burnout, já que possuem maior disponibilidade para o trabalho e dispõem de menos compromissos com tarefas domésticas, companheiros e filhos (PORTO AR, et al., 2013).

Referente à formação profissional dos entrevistados, foi verificado uma prevalência de técnicos de enfermagem, correspondendo a $43,8 \%$ dos entrevistados. Essa prevalência também foi verificada em outros estudos (SOUSA VFS e ARAUJO TCCF, 2015; CAMPOS ICM, et al., 2015; WORM FA, et al., 2016).

Nesse âmbito, estudos afirmam que os profissionais de enfermagem possuem grandes chances de desenvolvimento da síndrome de burnout por, dentre outros fatores, passarem mais tempo em contato com os pacientes e seus familiares, pelo grau de responsabilidade e precisão nos procedimentos, além de passarem diariamente por sofrimentos relacionados à morte, o que possibilita um maior risco de adoecimento (CAMPOS ICM, et al., 2015; WORM FA, et al., 2016).

Com relação à renda dos entrevistados, foi verificado uma codominância de até 2 salários mínimos $(39,7 \%)$ e de 3 a 5 salários mínimos (39,7\%). Em um estudo quantitativo, correlacional e de corte transversal, foi observado uma média de 1,38 salários mínimos ( $D P=1,09)$ (CAMPOS ICM, et al., 2015).

Um estudo estatístico, descritivo e inferencial realizado com profissionais de saúde, mostrou uma prevalência de 4 salários mínimos dentre os entrevistados, associando uma maior resiliência aos estresses laborais, dentre outros fatores, aos profissionais que possuem renda mensal superior a 7 salários mínimos (SOUSA VFS e ARAUJO TCCF, 2015). Outro trabalho associou a baixa renda mensal a fatores de insatisfação, estresse, esgotamento profissional e emocional, uma vez que esse processo se intensifica muitas vezes em decorrência de uma maior dedicação destes trabalhadores, a fim de compensar a baixa remuneração (WORM FA, et al., 2016).

Pesquisa com enfermeiros de uma UTI em São Paulo, identificou que a maioria dos profissionais que havia concluído o ensino médio apresentou burnout. Isso pode ser associado ao fato desses trabalhadores pertencerem a classes profissionais subordinadas, com salários mais baixos, em que há exigências e demandas, podendo influenciar no desenvolvimento da síndrome (FERNANDES LS, et al., 2017).

No tocante a carga horária de trabalho, uma pesquisa desenvolvida em três hospitais públicos e dois privados com 43 anestesiologistas em Maceió - Alagoas (AL) concluiu que nenhuma correlação foi vista entre carga horária e as dimensões de burnout, concordando com o resultado de outro estudo feito com médicos intensivistas de Maceió (BARBOSA FT, et al., 2017). Com isso, esse trabalho excessivo tanto está associado a SB como também aos erros médicos, pois viu-se que os profissionais com alta carga horária e/ou acometidos por SB tendem a errar mais (HOELZ L e CAMPELLO L, 2015).

Dessa forma, esse contexto é preocupante, já que muitos profissionais mantém uma jornada de trabalho excessiva para complemento da renda. Em um estudo com médicos intensivistas de cinco capitais brasileiras, observou-se que a maioria deles trabalhavam semanalmente entre 49 e 72 horas e mais de $20 \%$ dos responsáveis pelas UTI's adulto ultrapassavam 73 horas semanais (STAFFA TM, et al., 2016). 
Ademais, uma pesquisa com 182 profissionais de saúde de dois hospitais em Porto Alegre (RS), um privado e o outro público, apontou que os profissionais do serviço público apresentaram médias maiores em duas dimensões da síndrome de burnout: despersonalização e exaustão emocional comparado as médias do setor privado. Também foi encontrado que dos participantes 16, 3\% mantinham jornada de trabalho mais das 40 horas semanais no hospital público enquanto no privado somente $10 \%$ mantinham essa carga, tendo $74,7 \%$ dos profissionais trabalhando entre 30 e 40 horas por semana (SANTOS AS et al., 2017).

Em um estudo descritivo realizado com 60 médicos plantonistas de seis Unidades de Terapia Intensiva (UTI) da cidade de São Luís - Maranhão, foi identificado uma maior prevalência de burnout nos profissionais que trabalhavam em dois ou mais hospitais, assistiam mais de 10 pacientes por plantão, possuíam uma carga horária semanal menor que 60 horas, entretanto, com turno maior ou igual a 12 horas. Ao relacionar as condições de trabalho com a síndrome de burnout foi encontrada associação estatisticamente significativa entre o trabalho em UTI $(p=0,0004)$, trabalhar em mais de dois hospitais $(p=0,0095)$ e número de pacientes maior que 10 por plantão ( $p=0,0021$ ) (MARQUES GLC et al., 2018).

Um estudo descritivo e transversal realizado com 1225 enfermeiros de emergência da região de Andaluzia, na Espanha, confirma que os profissionais expostos a uma intensa jornada de trabalho possuem maiores chances de desenvolver SB. No presente estudo, foi evidenciado que $44,1 \%$ dos enfermeiros que realizam horas extras têm alta exaustão, contra $38 \%$ nos que não apresentam essa sobrecarga. Além disso, também houve diferença nas dimensões da SB, principalmente na exaustão emocional, no qual os profissionais expostos a maior carga horária apresentaram um índice de 15,3\% contra 10,8\% (CAÑADASDE LA FUENTE GA et al., 2016).

$\mathrm{Na}$ perspectiva do regime de trabalho, os profissionais diaristas apresentaram maior incidência da síndrome (15,5\%) em relação aos plantonistas (7\%). Resultados apontaram que 119 (84,40\%) dos profissionais entrevistados realizavam carga horária de 40 horas semanais e nove $(6,38 \%)$ pertenciam à escala de 30 horas semanais (FRANÇA FM, et al., 2012).

Um estudo realizado com 502 enfermeiros para analisar os fatores associados ao burnout com o seu respectivo turno de trabalho, evidenciou que os profissionais que trabalhavam no período noturno apresentavam níveis menores da SB (VIDOTTI V, et al., 2018). Enquanto os que exerciam sua função no período diurno demostraram maior probabilidade e os funcionários que trabalhavam em dois ou mais turnos apresentaram chances ainda maiores de adquirir a síndrome ( $p=0,031)$ (FERNANDES LS; et al., 2017). Apesar disso, outros autores mostraram não existir significância estatística entre o turno de trabalho e 0 burnout (MEDEIROS ARS, et al., 2019).

\section{CONCLUSÃO}

A importância deste estudo deu-se de forma a mensurar as dimensões do Burnout, ajudando a identificar os principais fatores de risco que estão diretamente ligados ao desencadeamento da síndrome nos profissionais da saúde. Através dele pode-se perceber que a doença tem um predomínio no sexo feminino variando na faixa etária dos 30 aos 39 anos, a maioria possui filhos, a categoria mais afetada entre os profissionais foram os técnicos de enfermagem, com mais de 10 anos de atuação na área, trabalhando 30 horas semanais e muito deles de maneira integral. A análise desses fatores de risco é importante para a saúde dos trabalhadores da área de saúde pois oferece subsídios para o seu reconhecimento e permite a adoção de medidas de prevenção e enfrentamento dessa problemática.

\section{REFERÊNCIAS}

1. BARBOSA FT, et al. Correlação entre a carga horária semanal de trabalho com a síndrome de Burnout entre os médicos anestesiologistas de Maceió -AL. Rev Bras Anestesiol. 2017; 67(2): 115-21.

2. BARROS MMS, et al. Síndrome de burnout em médicos intensivistas: estudo em UTls de Sergipe. Trends in Psychology / Temas em Psicologia. 2016; 24(1): 377-389.

3. BORGES EMN, et al. Compassion fatigue among nurses working on an adult emergency and urgent care unit. Rev. Latino-Am. Enfermagem. 2019; 27: e3175. 
4. CAMPOS ICM, et al. Fatores sociodemográficos e ocupacionais associados à síndrome de burnout em profissionais de enfermagem. Psychology/Psicologia Reflexão e Crítica. 2015; 28(4): 764-771.

5. CAÑADAS-DE LA FUENTE GA, et al. Risk factors and prevalence of burnout syndrome in the nursing profession. Int J Nurs Stud. 2015; 52(1): 240-249.

6. CAÑADAS-DE LA FUENTE GA, et al. Síndrome de Burnout em profissionais de enfermaria que realizam jornada física complementar em serviços de cuidados críticos e urgências. Rev. Espanhola de Saúde Pública. 2016; 90: e1-9

7. CORREDOR LH, et al. Bem-estar, esgotamento e segurança do pessoal da equipe de saúde: uma revisão sistemática. PLoS One . 2016; 11: e0159015.

8. ELDEVIK MF, et al. Insomnia, excessive sleepiness, excessive fatigue, anxiety, depression and shift work disorder in nurses having less than 11 hours in-between shifts. PLoS One. 2013; 8(8): e70882.

9. FERNANDES LS, et al. Síndrome de burnout em profissionais de enfermagem de uma unidade de terapia intensiva. Rev Pesqui Cuid Fundam. 2017; 9(2): 551-557.

10. FRANÇA FM, et al. Burnout e os aspectos laborais na equipe de enfermagem de dois hospitais de médio porte. Rev Latino-Am Enfermagem. 2012; 20(5): 961-970.

11. GARCIA CL, et al. Influência do Burnout na segurança do paciente: revisão sistemática e meta-análise. Medicina (Kaunas). 30 de agosto de 2019; 55 (9): 553.

12. GIL-MONTE PR, et al. Validação de versão brasileira do "Cuestionario para la Evaluación del Síndrome de Quemarse por el Trabajo" em professores. Rev Saúde Pública 2010; 44(1): 140-7.

13. GRILLO LP, et al. Fatores de risco e proteção para o desenvolvimento de doenças crónicas em profissionais de enfermagem. Revista de Enfermagem Referência. 2018; 4(18): 63-72.

14. HOELZ L, CAMPELLO L. Relação entre Síndrome de Burnout, erro médico e longa jornada de trabalho em residentes de medicina. Rev Bras Med Trab. 2015; 13(2): 126-34.

15. LEITER MP, MASLACH C. Latent burnout profiles: A new approach to understanding the burnout experience. Burn Res. 2016; 3(4): 89-100.

16. LIMA RAS, et al. Vulnerabilidade ao burnout entre médicos de hospital público do Recife. Cien Saude Colet 2013; 18(4):1051-1058.

17. MAGALHÃES E, et al. Prevalência de síndrome de burnout entre os anestesiologistas do Distrito Federal. Rev. Bras. Anestesiol. $2015 \mathrm{abr}$; 65(2): 104-110.

18. MARQUES GLC, et al. Síndrome de burnout entre médicos plantonistas de unidades de terapia intensiva. J. bras. psiquiatr. 2018;67(3): 186-193.

19. MASLACH C, LEITER MP. Understanding the burnout experience: recent research and its implications for psychiatry. Wld Psychiatry. 2016; 15(2): 103-11.

20. MEDEIROS ARS, et al. O burnout em profissionais de enfermagem que atuam em um complexo psiquiátrico. Rev. Enferm. UFSM, Santa Maria. 2019; 9(e36): 1-16.

21. OPAS. Organização Pan-Americana de Saúde. CID: burnout é um fenômeno ocupacional. Brasília, DF 2019.

22. PERNICIOTTI P, et al. Síndrome de Burnout nos profissionais de saúde: atualização sobre definições, fatores de risco e estratégias de prevenção. Rev. SBPH. 2020; 23(1): 35-52.

23. PORTO AR, et al. Autoavaliação de saúde e doenças crônicas entre enfermeiros de Pelotas/RS. Revista Eletrônica de Enfermagem. 2013; 15(3): 763-771.

24. ROSEINSTEI AH, Physician Stress and Burnout: Prevalence, cause and Effect. AAOS Now. [Internet]. 2012.

25. SANTOS AS, et al. Contexto hospitalar público e privado: impacto no adoecimento mental de trabalhadores da saúde. Trab. educ. saúde. 2017; 15(2): 421-38.

26. SANTOS MA, HORMANEZ M. Atitude frente à morte em profissionais e estudantes de enfermagem: revisão da produção científica da última década. Ciência \& Saúde Coletiva [online]. 2013, 18(9): 2757-2768.

27. SCHEFFER, M. et al. Demografia Médica no Brasil 2018. São Paulo, SP: FMUSP, CFM, Cremesp, 2018.286 p. ISBN: 978-85-87077-55-4

28. SILVA SCPS, et al. A síndrome de burnout em profissionais da Rede de Atenção Primária à Saúde de Aracaju, Brasil. Ciência \& Saúde Coletiva. 2015; 20(10): 3011-3020.

29. SOUSA, VFS, ARAUJO TCCF. Estresse Ocupacional e Resiliência Entre Profissionais de Saúde. Psicologia: Ciência e Profissão. 2015; 35(3): 900-915.

30. STAFFA TMO, et al. Prevalência de síndrome de burnout em médicos intensivistas de cinco capitais brasileiras. Rev bras ter intensiva. 2016; 28(3): 270-77.

31. STAMM BH. The Concise ProQOL Manual. 2nd ed. Pocatello, ID: ProQOL.org. 2010. Disponível em: https://proqol.org/uploads/ProQOLManual.pdf. Acesso em: 24 mar. 2019.

32. OIT. Organización Internacional del Trabajo. Estrés en el trabajo. Un reto colectivo. Ginebra; OIT: 2016

33. URBANETTO JS, et al. Workplace stress in nursing workers from an emergency hospital: Job Stress Scale analysis. Rev. Latino-Am. Enfermagem. 2011; 19(5): 1122-1131.

34. VIDOTTI V, et al. Burnout Syndrome and shift work among the nursing staff. Rev. Latino-Am. Enfermagem. 2018; 26: e3022.

35. VIEIRA I, RUSSO JA. Burnout e estresse: entre medicalização e psicologização. Physis: Revista de Saúde Coletiva [online]. 2019; 29(2): 1-22.

36. WORM FA, et al. Risco de adoecimento dos profissionais de enfermagem no trabalho em atendimento móvel de urgência. Rev Cuid. 2016; 7(2): 1288-1296. 